\title{
LONGITUDINAL BROADBAND IMPEDANCE MEASUREMENT SYSTEM BY COAXIAL LINE METHODS *
}

\author{
Huang Gang, Huang Wenhui, Tong Dechun, Tsinghua University, Beijing, P.R.China \\ Zhao Zhentang, Shanghai Synchrotron Radiation Canter, Shanghai P.R.China
}

\begin{abstract}
Impedance of vacuum components in the ring is very important in the analysis of beam instability. This paper describes the construction of a broadband impedance measurement system. This system applies the Coaxial line methods of M.Sands and J.Rees. The system is made up of HP8720B vector network analyser and a PC computer. A new $\mathrm{RF}$ connector is designed to get the inner conductor straining and RF impedance matching. A pillbox cavity is measured by this system. And the result was agreed with MAFIA calculation well.
\end{abstract}

\section{INTRODUCTION}

Impedance of vacuum components in the ring is very important in the design of an accelerator. The impedance of different components can be estimated by analysis, calculation, or bench measurement during the design stage.

Coaxial Line Method is a typical bench measurement method in measuring impedance, which is first

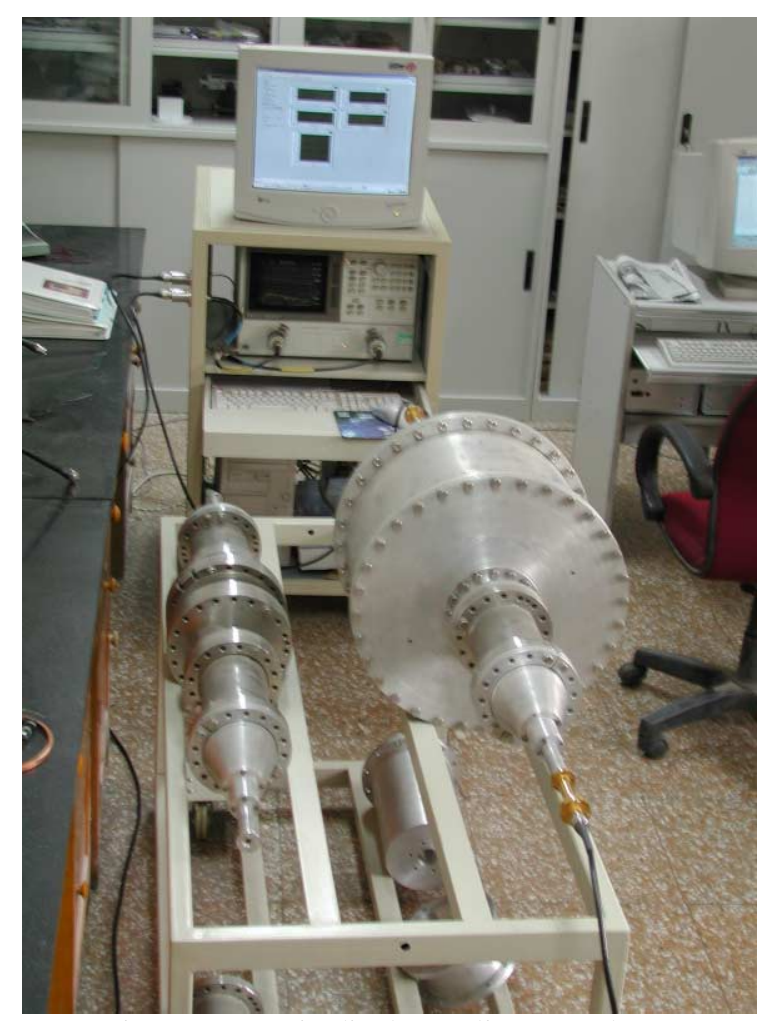

Figure 1: Longitudinal Coupling Impedance Measurement System developed by Sands and Rees ${ }^{[1]}$. After that, several laboratory construct their own measurement platform. This method uses an inner conductor to simulate the bunch behaviour. The inner conductor forms a coaxial system together with the Device Under Test(DUT) or reference tube. Measuring the $S$ parameter of such coaxial systems $S_{21, \text { REF }}$ and $S_{21 \text { DUT, we can get the }}$ coupling impedance $\mathrm{Z}(\mathrm{w})$ by the following relationship ${ }^{[2][3]}$ :

$$
Z_{w}=2 R_{0} \frac{S_{21, R E F}-S_{21, D U T}}{S_{21, D U T}}
$$

where $\mathrm{R}_{0}$ is characteristic impedance of transmission line.

Traditional measurement is done in time domain. The measurement platform is made up of synthesis pulse generator and oscillograph. With the development of instrument, nowadays, people do the measurement in frequency domain by vector network analyser.

Such a longitudinal impedance measurement system is constructed in Tsinghua University. The system including a HP8720B vector network analyser, a GPIB card, a personal computer and software written by LabView, together with related microwave measurement components, as shown in Figure 1.

The relationship between coupling impedance and $\mathrm{S}$ parameter shown in formula (1) is applicable only when the whole microwave system outside DUT are matching transmission, and the inner conductor is thin enough and strained.

A special RF connector is designed to accomplish the request above. Measurement of a pillbox Cavity is done to calibrate the system.

\section{SPECIAL CONNECTOR DESIGN ${ }^{[4]}$}

\subsection{Requirement}

The RF connector should be able to accomplish following requirement:

- It should have a good transmission parameters among wide frequency range. The transmission factor will related to the signal to noise directly.

- It should be able to straining inner conductor. The inner conductor should be stained to keep it straight.

- It should have a good repeatability. The transmission factor of the DUT and reference tube will be measured separately. The connector

*Supported by National Natural Science Foundation of China 19875065 


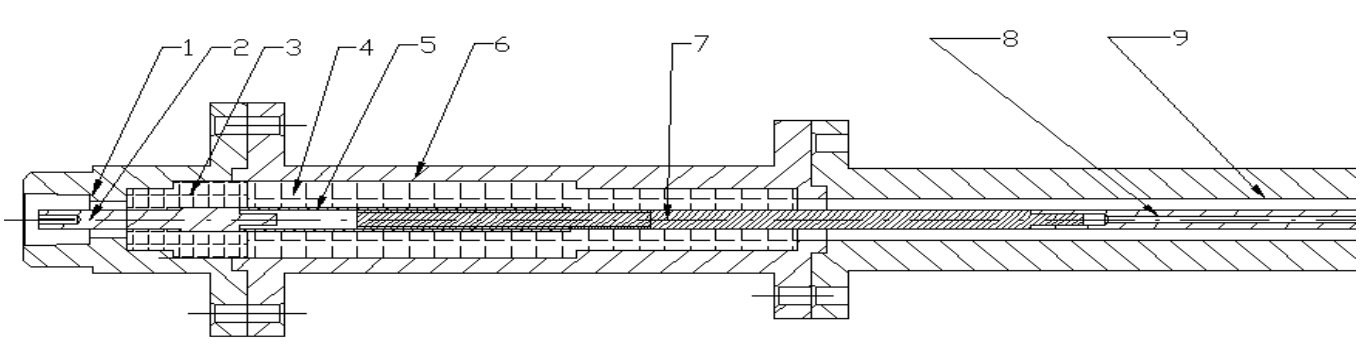

(a)

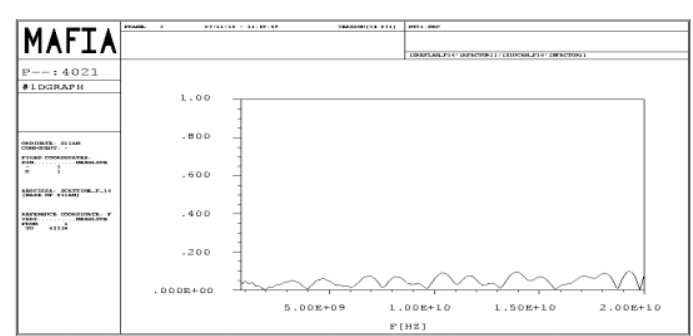

(b)

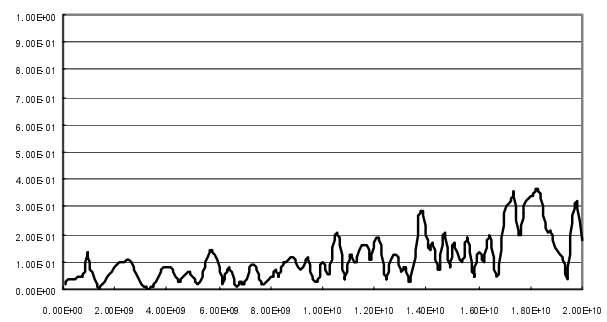

(c)

Figure 2: (a). Mechanism Design of the connector (b). Calculated $\mathrm{S}_{11}$ by MAFIA (c). Measured $\mathrm{S}_{11}$ of the connector

should keep the same performance between the measurements of $S_{21, \text { REF }}$ and $S_{21, \text { DUT }}$.

\subsection{Design and Measured Result of the connector}

MAFIA is used to design the special connector. A structure with two discontinuity is applied in the connector, as shown in Figure 2-a. The connector is designed with a movable inner conductor which straining the thread of the measurement system by screw thread. Such a design makes the electronic boundary of the connector constant during straining process.

The calculated $S_{11}$ of the connector is less then 0.1 below 20GHz, as shown in Figure 2-b. The connector is manufactured and measured with network analyzer. The measure performance of the connector is shown in Figure 2-c. $S_{11}$ of the connector is less then 0.2 below $17 \mathrm{GHz}$ with only some point exception.

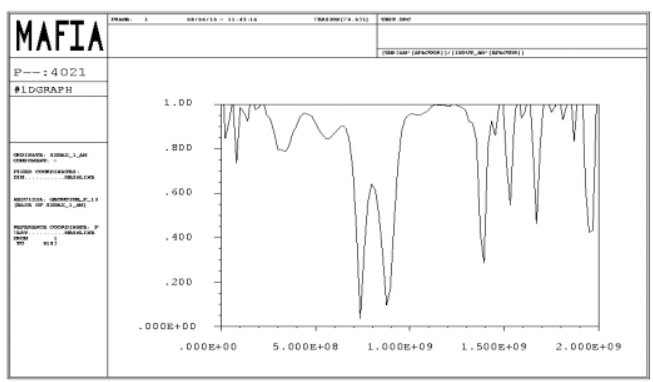

(a)

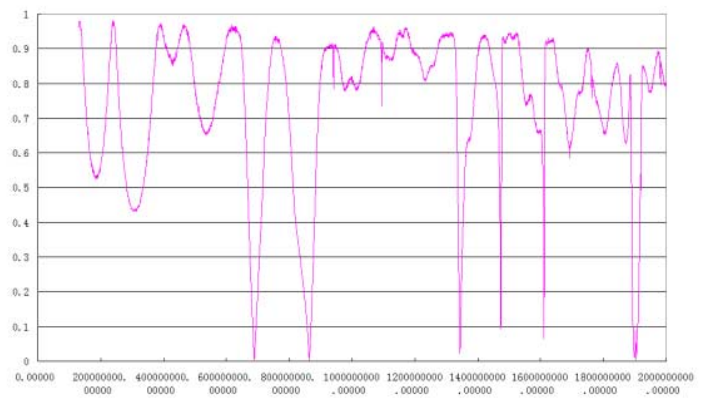

(c)

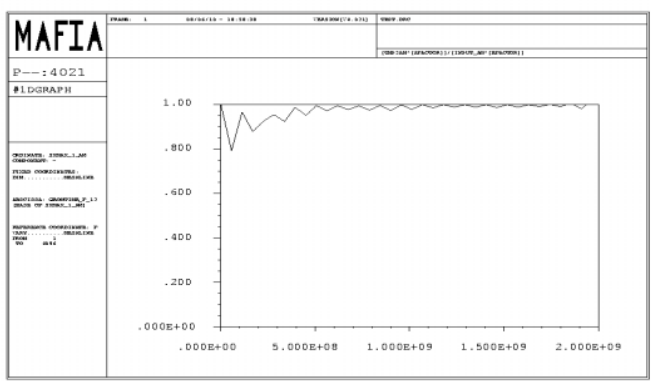

(b)

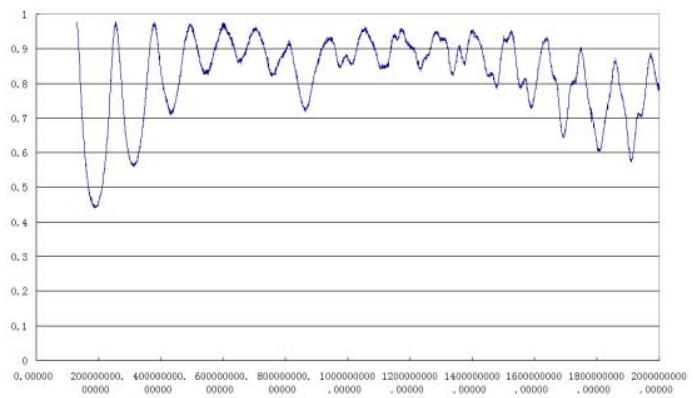

(d)

Figure 3: (a) Calculated $S_{21}$ of the system with pillbox (b) Calculated $S_{21}$ of the system with reference tube (c) Measured $S_{21}$ of the system with pillbox (d) Measured $S_{21}$ of the system with reference tube 


\section{MEASUREMENT OF A PILLBOX CAVITY}

Typically, coaxial line method is used to measure broadband longitudinal coupling impedance of low Q components, such as bellows, separator etc. In order to check the availability of the measurement system, some examples can be calculated should be measured.

As a simple example of the longitudinal coupling impedance measurement, a pillbox cavity is measured and the result is compared with MAFIA calculation.

The calculation models include the pillbox cavity or the reference tube, two drift sections, two tapered matching sections. The calculated $S_{21}$ is shown in Figure $3-a$ and 3-b. It is shown that there is slightly reflection in the lower frequency range when the reference tube is connected. And when the pillbox cavity is connected into the measurement system, there are much reflection at the frequency of modes of the cavity. Same result can be achieved from measurement as shown in Figure 3-c and 3-d. There are several coaxial modes separated about $150 \mathrm{MHz}$. They all can be subtracted from the measurement of reference tube. The difference of valley values is come from the reflection of the connector.

The impedance of the pillbox cavity can be calculated by formula (1) with the data of measured $S_{21}$. The calculated result is shown in figure 4.

Modes of the pillbox cavity can also be calculated by E module of MAFIA. Modes of the pillbox with and without central line are listed in the follow table:

\begin{tabular}{|c|c|c|}
\hline Modes(MHz) & With central line & Without central line \\
\hline 1 & 674.694 & 607.158 \\
\hline 2 & 927.381 & 869.255 \\
\hline
\end{tabular}

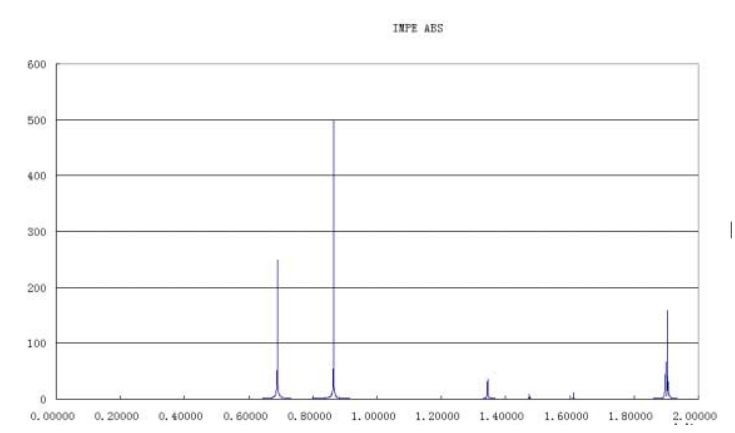

Figure 4: calculated impedance of the pillbox cavity

\section{FURTHER DEVELOPMENT}

The tapered matching section used now is circular to $\mathrm{N}$ type connector size. Two extra tapers are needed to convert the cross section from vacuum chamber to circle if we want to measure other vacuum components around the ring, A new tapered matching section is designed to fit vacuum chamber of IHEP and SSRC. The taper is designed with double cosine function as transmit function. And is now fabricating by the CIMS center of
Tsinghua University. Figure 5 is quarter of the inner surface of the taper.

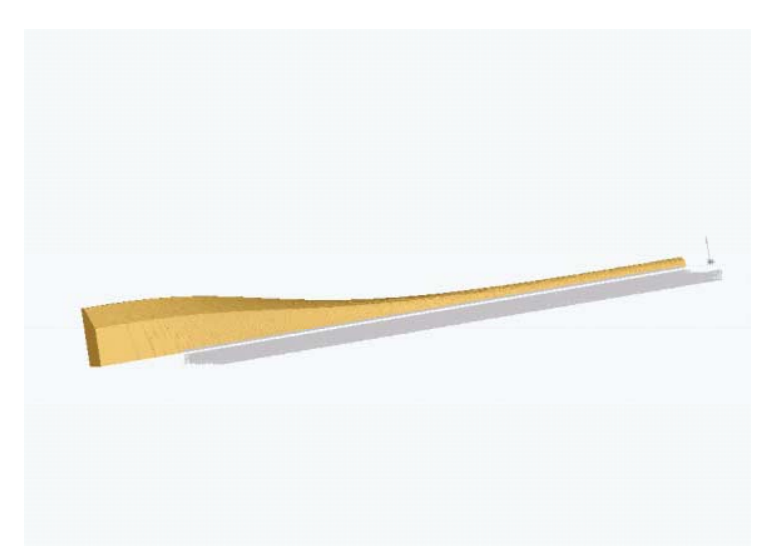

Figure 5: quarter of the inner surface of the new taper.

HP 8720B standard calibration of the vector network analyser is now used in the measurement. This method can only correct the error outside the connector. Accuracy of the measurements requires correct all errors, including tapered matching section, drift tube section. Through Short Delay calibration is to be used in the measurement system. With this calibration, calibrated plane can be moved to the entrance of the DUTs of references.

\section{RESULT}

- A longitudinal impedance measurement system is constructed.

- A new type of RF broadband connector is designed and fabricated.

- The measurement of pillbox cavity proved the practicable of the impedance measurement system.

- A new tapered matching section is designed and being fabricated. With this taper, more vacuum components can be measured by the system.

- TSD calibration is to be used in the measurements.

\section{REFERENCES}

[1] M.Sands J.Rees, "A Bench Measurement of the Energy Loss of a Stored Beam to a Cavity," PEP-95, 1974.

[2].H.Hahn F.Pedersen, "On Coaxial Wire Measurements of the Longitudinal Coupling Impedance," BNL-50870, April 1978.

[3] A.Argan L.Palumbo M.R.Masullo V.G.Vaccaro, "On the Sands and Rees Measurement Method of the Longitudinal Coupling Impedance," PAC'99 1999

[4] Huang Gang Huang Wenhui Chen Huaibi Zheng Shuxin Tong Dechun Lin Yuzheng Zhao Zhentang, "Developing a Special RF Connector for Coaxial Impedance Measurement Platform" to be published 